\title{
Comparison of the pathologic and pathogenic features in six different regions of postmortem brains of three patients with fatal familial insomnia
}

\author{
WU-LING XIE ${ }^{1,2^{*}}$, QI SHI ${ }^{2 *}$, SHENG-LI XIA ${ }^{3 *}$, BAO-YUN ZHANG ${ }^{2}$, HAN-SHI GONG $^{1,2}$, \\ SHAO-BIN WANG ${ }^{2}$, YIN XU $^{2}$, YAN GUO $^{2}$, CHAN TIAN $^{2}$, JIN ZHANG $^{2}$, \\ BIAN-LI XU ${ }^{3}$, YONG LIU ${ }^{1}$ and XIAO-PING DONG ${ }^{2,4}$
}

\begin{abstract}
${ }^{1}$ School of Medicine, Xi'an Jiao Tong University, Xi'an, Shaanxi 710061; ${ }^{2}$ State Key Laboratory for Infectious Disease Prevention and Control, National Institute for Viral Disease Control and Prevention, Chinese Center for Disease Control and Prevention, Beijing 102206; ${ }^{3}$ Henan Provincial Center for Disease Control and Prevention, Zhengzhou, Henan 450016; ${ }^{4}$ Key Laboratory of Pathogenic Microbiology and Immunology, Institute of Microbiology, Chinese Academy of Sciences, Beijing 100101, P.R. China
\end{abstract}

Received August 3, 2012; Accepted October 5, 2012

DOI: $10.3892 /$ ijmm.2012.1194

\begin{abstract}
Fatal familial insomnia (FFI) is an autosomal dominant prion disease clinically characterized by rapidly progressive insomnia, prominent autonomic alterations and behavioral disturbance. The D178N mutation of the prion protein gene (PRNP) on chromosome 20 in conjunction with methionine at codon 129 is a molecular feature. Although the neuropathological characteristics of FFI are well documented, the neuropathologic and pathogenic features of FFI patients remain poorly understood. Six brain regions of postmortem brains from 3 FFI patients were examined using immunohistochemistry, western blot analyses and quantitative real-time PCR. In all 3 brain specimens, reactive astrogliosis was found to be more severe in the thalamus than in the cortex regions. Western blot analyses showed that all three brains expressed PrP, but only 2 were associated with significantly weak proteinase $\mathrm{K}(\mathrm{PK})$ resistance. However, the conformational stabilities of $\mathrm{PrP}^{\mathrm{Sc}}$ in the 3 FFI brains were significantly weaker than those presented in a G114V genetic Creutzfeldt-Jakob disease (gCJD) case. Immunohistochemistry and western blot analyses showed comparable amounts of neuron-specific enolase (NSE)-positive stained cells and NSE protein among the different regions in the three brains. In addition, the transcriptional levels of glial fibrillary acidic protein
\end{abstract}

Correspondence to: Professor Xiao-Ping Dong, State Key Laboratory for Infectious Disease Control and Prevention, National Institute for Viral Disease Control and Prevention, Chinese Center for Disease Control and Prevention, Chang-Bai Road 155, Beijing 102206, P.R. China

E-mail: dongxp238@sina.com

Professor Yong Liu, School of Medicine, Xi'an Jiao Tong University, Xi'an, Shaanxi 710061, P.R. China

E-mail: liuy5599@mail.xjtu.edu.cn

${ }^{*}$ Contributed equally

Key words: prion, astrogliosis, neuron loss, glial fibrillary acidic protein, neuron specific enolase
(GFAP) and NSE-specific mRNAs were coincident with the expression of these proteins. In conclusion, in the present study, we described the detailed regional neuropathology of FFI cases.

\section{Introduction}

Human prion diseases, also known as transmissible spongiform encephalopathies (TSEs), are fatal neurodegenerative disorders including Kuru, Creutzfeldt-Jakob disease (CJD), GerstmannSträussler-Scheinker disease (GSS) and fatal familial insomnia (FFI). As an autosomal dominant prion disease, FFI patients carry the D178N mutation coupled with a methionine at the polymorphic site 129 of the prion protein gene (PRNP) on chromosome $20(1,2)$. Clinically, FFI is characterized by rapidly progressive insomnia, prominent autonomic alterations and behavioral disturbance $(3,4)$. The prominent pathology of FFI occurs mainly in the thalamus, showing severe astrogliosis and loss of neurons, however, the lesion profiles may vary in different patients and different brain areas $(3,5,6)$. Additionally, deposits of $\mathrm{PrP}^{\mathrm{Sc}}$, the pathological isoform of $\mathrm{PrP}$, in brain tissues of FFI are usually infrequent $(2,7)$.

More than ten FFI patients have been diagnosed genetically and/or pathologically since conducting CJD surveillance in mainland China, starting in 2006. To further investigate the neuropathologic and pathogenic features of Chinese FFI patients, postmortem brains from three FFI patients were comparatively analyzed based on $\mathrm{PrP}^{\mathrm{Sc}}$, the formation of astrogliosis and the loss of neurons in various brain regions. Meanwhile, the transcriptions of glial fibrillary acidic protein (GFAP) and neuron-specific enolase (NSE) specific mRNA in different regions were evaluated. Despite the different status of $\mathrm{PrP}^{\mathrm{Sc}}$ deposits in brains among the three patients, all cases showed more severe reactive astrogliosis in the region of the thalamus than in the cortex region. Neuronal loss among different regions was highly comparable. We also observed that the transcriptional levels of GFAP and NSE specific mRNAs were coincident with the expressions of respective proteins in brain tissues of the FFI patients. 


\section{Materials and methods}

Patients. The postmortem brains of three FFI cases that were diagnosed by the experts from the Chinese National Surveillance Network for CJD (CNSNC) were enrolled in this study. The clinical presentation and pedigree of the family for 2 of the 3 cases, a 48 -year-old male (Case 1) and a 26-year-old female (Case 2), were described previously (8).

Case 3 was a 55-year-old man who was referred to the first affiliated hospital of Zhengzhou University in 2009. The patient was hospitalized with complaints of sleep disturbance and restlessness for more than 1 month. During the initial admission, the patient complained of general tiredness and weakness, accompanied by belching and sour regurgitation. Neurological examination showed mutism, generalized hyperreflexia of the limbs and bilateral Babinski signs (+). During hospitalization, physical movements rapidly declined and the patient later became bedridden. EEG examination did not suggest CJD-related electroencephalogram periodic sharp wave complexes (PWSC) and MRI scanning did not reveal any obvious abnormalities. CSF 14-3-3 protein was positive. His general condition deteriorated progressively and he was discharged 4 months later. Severe akinetic mutism marked the end of his clinical course and the patient succumbed to the disease 2 months after discharge. The total clinical course was approximately 8 months. His peripheral blood was obtained when he was in hospital, and PRNP gene sequencing revealed a D178N mutation with M129M homozygote.

Interviews with this patient's family members revealed that an elder brother had also developed similar symptoms at the age of 55. The symptoms included initial insomnia and restlessness, followed by progressive dementia, severe myoclonus, pyramidal and extrapyramidal signs. Akinetic mutism, sleepiness, lethargy marked the terminus of the clinical course which lasted 14 months. Eleven family members of the Case 3 patient were screened for PRNP. D178N mutations were observed in his second elder brother and the son of this brother who was still healthy. The families of the other patients did not consent to genetic analysis. The pedigree of the family of Case 3 is shown in Fig 1. The main clinical manifestations of the 3 FFI cases are summarized in Table I.

Preparation of brain homogenates and western blot analyses. Human brain tissues from the regions of the thalamus (mediodorsal thalamic nuclei), the cingulate gyrus, the frontal cortex, the parietal cortex, the occipital cortex and the temporal cortex of each patient, brain tissues of a definitely diagnosed G114V genetic CJD (gCJD) patient and a sporadic CJD (sCJD) patient, as well as normal C57BL/6 mouse brains, were individually collected. Brain homogenates $[10 \%(\mathrm{w} / \mathrm{v})]$ were prepared based on the protocol previously described (8). Aliquots of brain homogenates were separated on 12\% SDS-PAGE and electroblotted onto a nitrocellulose membrane. Membranes were probed with various primary antibodies at $4^{\circ} \mathrm{C}$ overnight, including 1:2,000-diluted anti-neuron specific enolase mAb (NSE; Abcam, UK), 1:1,000-diluted anti-glial fibrillary acidic protein mAb (GFAP; Santa Cruz Biotechnology, Inc., Santa Cruz, CA, USA), 1:5,000-diluted anti-PrP mAb (3F4; Chemicon) and 1:1,000-diluted anti- $\beta$-actin mAb (Santa Cruz Biotechnology, Inc.), respectively. After incubating with

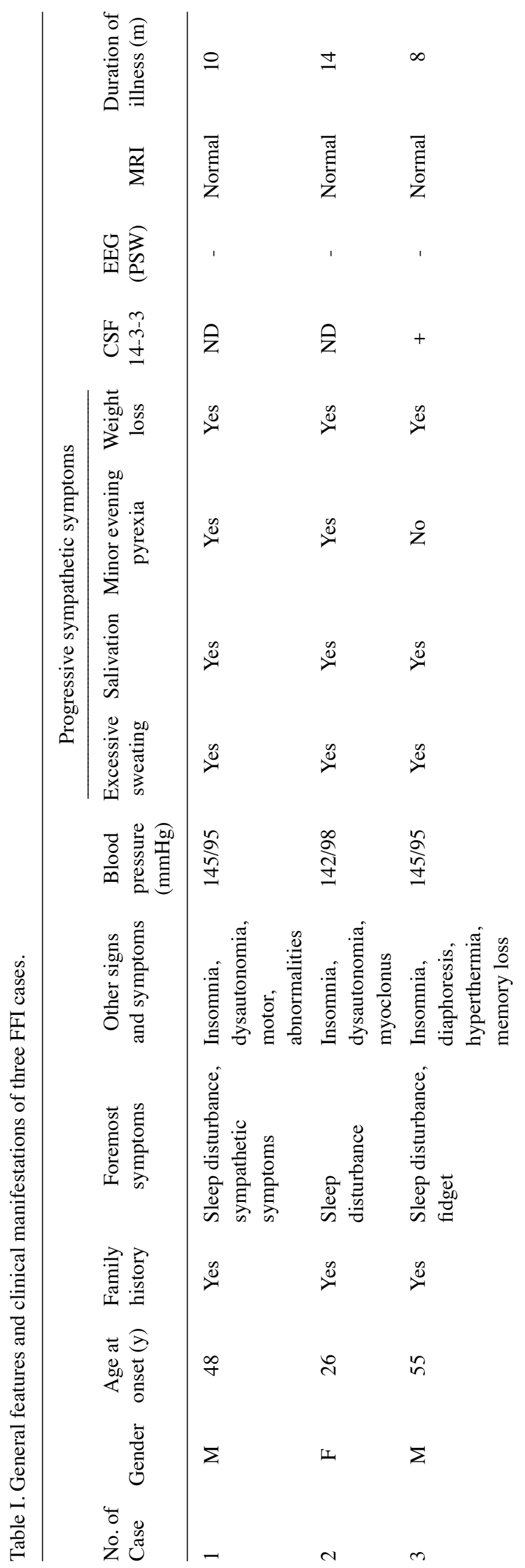




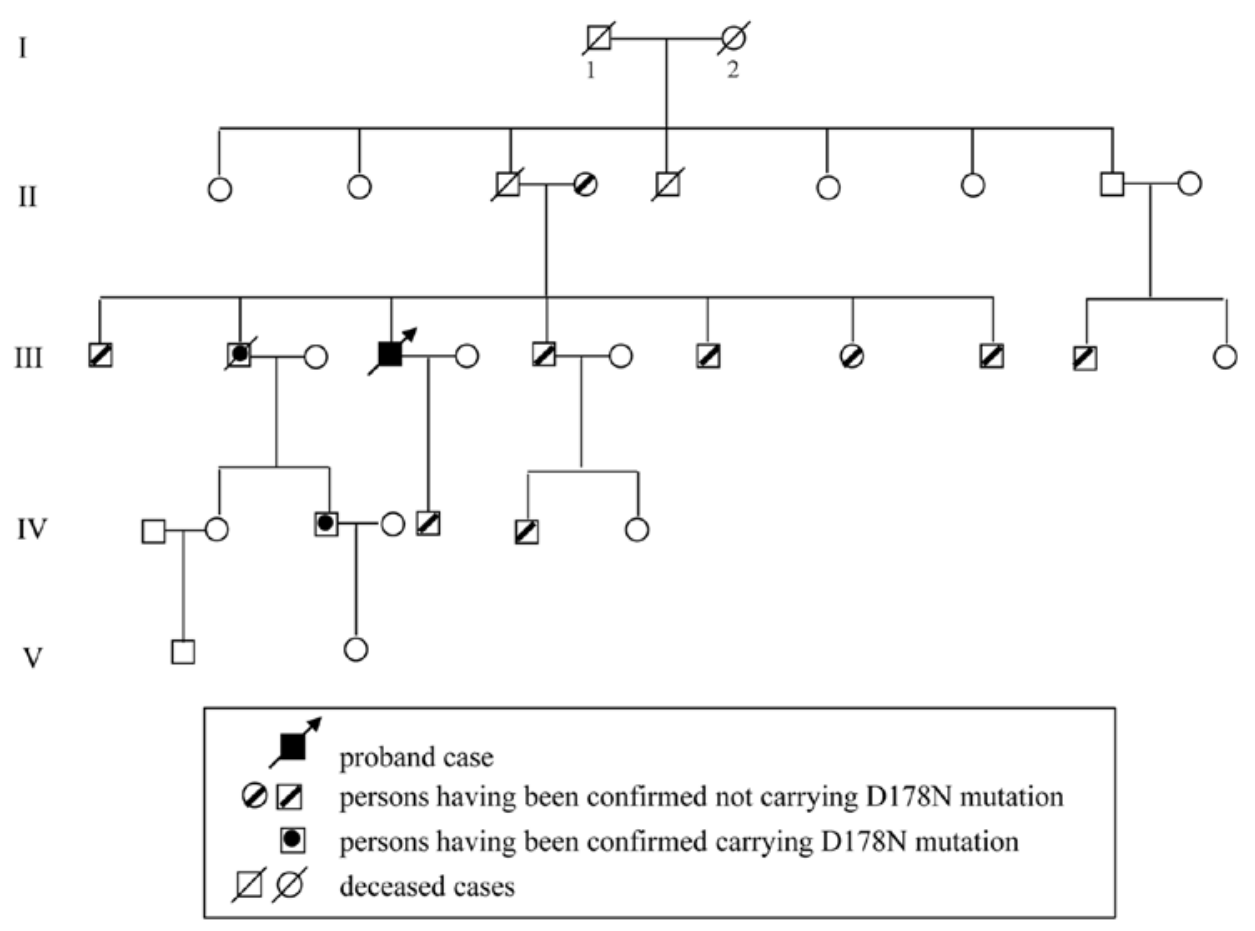

Figure 1. Pedigree of the FFI Case 3 family. The pedigree of the proband's lineage comprising 33 members across 5 generations.

1:5,000-diluted horseradish peroxidase (HRP)-conjugated goat anti-mouse IgG (Santa Cruz Biotechnology, Inc.) at room temperature for $2 \mathrm{~h}$, the blots were developed using Enhanced Chemiluminescence system (ECL; Amersham Life Sciences) and visualized on autoradiography films. Images were captured by ChemiDoc ${ }^{\mathrm{TM}}$ XRS+ Imager (Bio-Rad) and analyzed with Image J software.

To detect the presence of proteinase $\mathrm{K}(\mathrm{PK})$ resistant $\mathrm{PrP}$ in brain tissues, the brain homogenates were digested with a final concentration of $20 \mu \mathrm{g} / \mathrm{ml} \mathrm{PK}$ at $37^{\circ} \mathrm{C}$ for $60 \mathrm{~min}$ prior to western blot analyses. To compare the PK-resistance of PrP among the 3 brains, tissue homogenates from the thalamus were subjected to treatment of various amounts of PK, i.e. 0,5 , $10,15,20$ and $25 \mu \mathrm{g} / \mathrm{ml} \mathrm{PK}$.

Conformational stability assay. One hundred microliters of tissue homogenates of the thalamus region from each FFI case, a G114V gCJD patient (9) and an SCJD case, were mixed with 5 volumes of cold methanol at $-20^{\circ} \mathrm{C}$ for $2 \mathrm{~h}$. Following centrifugation at $20,000 \mathrm{x}$ g for $30 \mathrm{~min}$, the pellets were resuspended in $100 \mu \mathrm{l} \mathrm{GdnHCl}(1,2,3,4,5$ and $6 \mathrm{M})$ and incubated at $37^{\circ} \mathrm{C}$ for $12 \mathrm{~h}$ as described elsewhere (10). Subsequently, five volumes of cold methanol was added into each preparation for protein precipitation and the pellets were resuspended with $100 \mu \mathrm{l}$ TN buffer (10 mM Tris, $130 \mathrm{mM} \mathrm{NaCl,} \mathrm{pH} \mathrm{7.0).}$ Half the section was employed directly into SDS-PAGE and the rest was subjected to PK-digestion $(20 \mu \mathrm{g} / \mathrm{ml})$ prior to SDS-PAGE.

Nissl staining and immunohistochemical (IHC) assays. Brain paraffin sections $(5 \mu \mathrm{m})$ of 6 brain regions were prepared. For Nissl staining, slices were stained with Nissl (1\% toluidine blue) for $30 \mathrm{~min}$ based on the protocol described elsewhere
(11). IHC assays were performed according to published protocol (12). Briefly, the sections were incubated overnight at $4^{\circ} \mathrm{C}$ with 1:500-diluted mAb for NSE, or 1:500-diluted mAb for GFAP. Then, the sections were incubated for $1 \mathrm{~h}$ with 1:250-diluted HRP-conjugated goat anti-mouse secondary antibody (Vector Laboratories, USA) and visualized by incubation with 3,3-diaminobenzidine tetrahydrochloride (DAB). GFAP or NSE-positive staining regions were separately assessed based on integral optical density (IOD) representing the total intensity of all positive signals in an optical field (13). The OD analyses were performed in 3 individual sections by 2 investigators under double blindness. Nonspecific background correction in each section was carried out by subtracting the OD value of the blank area obtained from the same section.

Quantitative real-time PCR ( $q R T-P C R)$. Real-time PCR in this study was performed in an ABI $7900 \mathrm{HT}$ Fast sequence detector (Applied Biosystems). Total tissue RNAs were extracted from different brain regions with a commercial RNeasy Lipid Tissue mini kit (Qiagen), and were subjected to first-strand cDNA synthesis with Reverse Transcription System (Promega) according to the manufacturer's protocols. The specific primers were designed based on the sequences of human GFAP, NSE and GAPDH in GenBank (NM_183079.1, NM_001975.2 and NM_002046.3), including: 5'-GGAGA GGAAGATTGAGTCGC-3' and 5'-CGGTAGTCGTTGG CTTCG-3', 5'-CGACAAGGCTGGCTACAC-3' and 5'-CAG GTCATCACCCACAATC-3', and 5'-ACCACAGTCCAT GCCATCAC-3' and 5'-TCCACCACCCTGTTGCTGTA-3', respectively. PCR amplification was performed in triplicate with a total of 40 cycles $\left(15 \mathrm{sec}\right.$ at $95^{\circ} \mathrm{C}$ and $30 \mathrm{sec}$ at $56^{\circ} \mathrm{C}$ ). The comparative $\mathrm{Ct}$ (the fractional cycle number at which the 


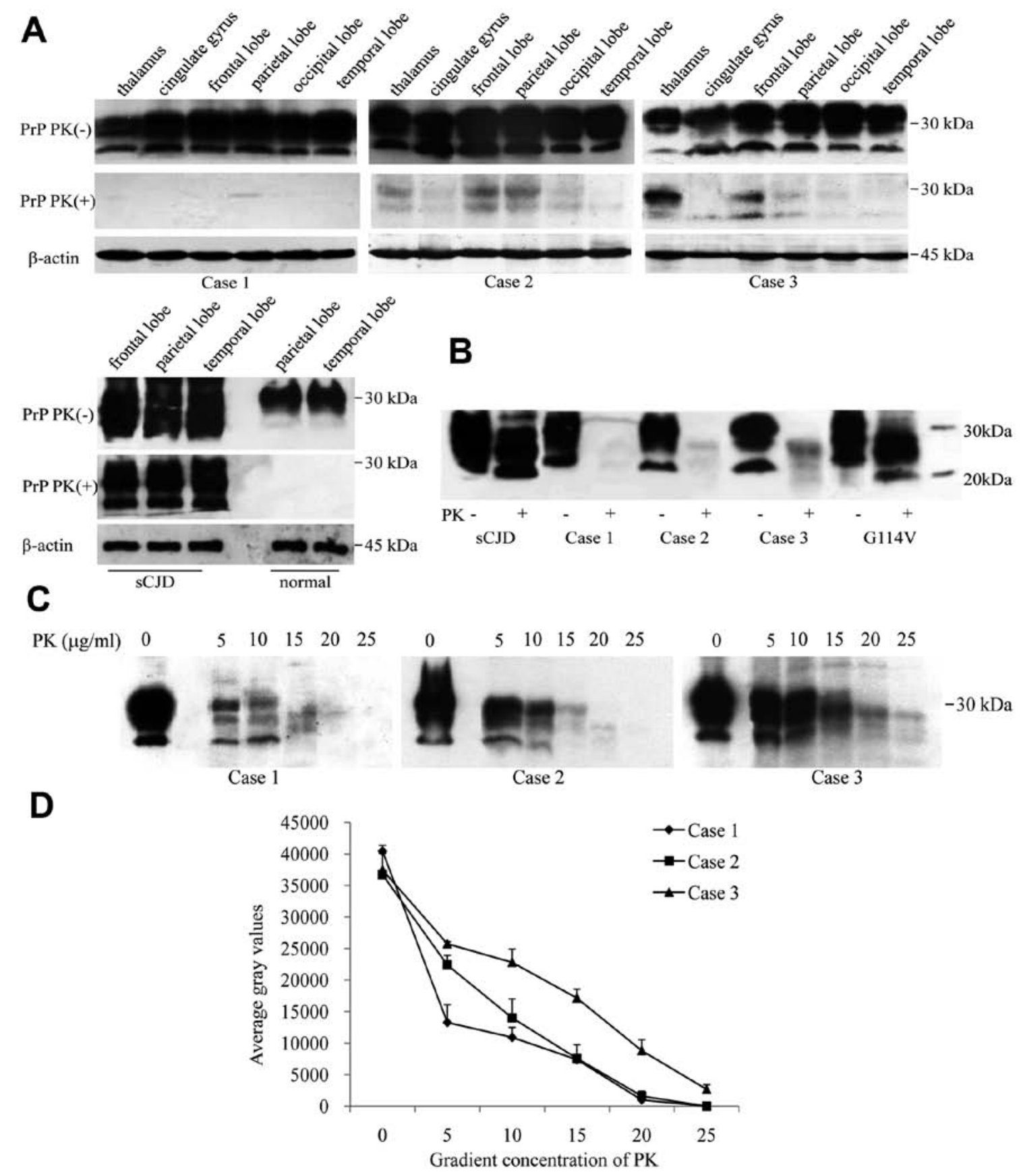

Figure 2. Presence of $\mathrm{PrP}^{\mathrm{Sc}}$ in the various brain regions of the 3 FFI cases. (A) Western blot analyses. The immunoblots for total PrP (without PK treatment), $\mathrm{PrP}^{\mathrm{Sc}}$ (digested with $20 \mu \mathrm{g} / \mathrm{ml} \mathrm{PK}$ ), and $\beta$-actin are shown in the upper, middle and lower parts, respectively. Brain tissues of an sCJD and a healthy human are used as controls. (B) The immunoblots show the $\mathrm{PrP}^{\mathrm{Sc}}$ patterns among 3 FFI cases, an SCJD and a G114V gCJD. (C) Comparative analyses of the PK-resistances of $\mathrm{PrP}$ in the regions of the thalamus. (D) Quantitative analyses of each gray numerical value of $\mathrm{PrP}^{\mathrm{Sc}}$ blots after treatment with a serial concentration of PK.

amount of amplified target reached a fixed threshold) method was used for the relative quantitative detection of the expressions of the targeting genes. The relative $\mathrm{Ct}$ for the target gene was subtracted from the $\mathrm{Ct}$ for the GAPDH gene (14).

Statistical analysis. Statistical analyses were performed using SPSS 17.0 statistical package. All values in figures were expressed as means \pm SD. Quantitative analysis of immunoblot images was carried out using software ImageJ. Differences between means were assessed with the one-way ANOVA test. P-value $<0.05$ was considered to indicate statistically significant differences.
Ethics statement. Written consent for further investigation and publication was obtained from the relatives of the patients. Usage of the stored human brain samples in this study was approved by the Ethics Committee of the National Institute for Viral Disease Prevention and Control, China CDC.

\section{Results}

Features of $\operatorname{PrP}^{S c}$ in FFI cases. Six brain regions of each patient were comparatively analyzed with PrP-specific western blot analyses. The signals of three typical glycosylated isoforms of PrP were observed in all preparations without 
A

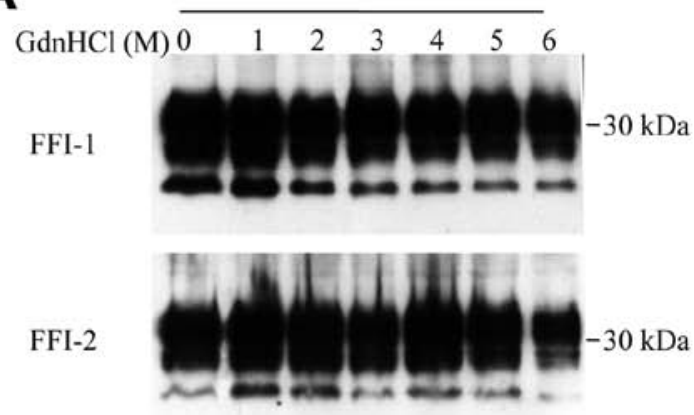

FFI-3

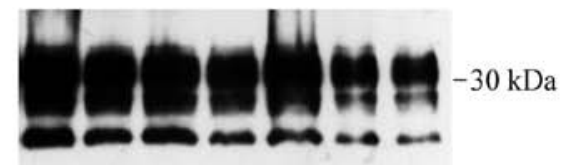

G114V

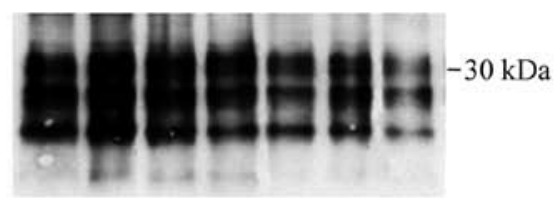

sCJD

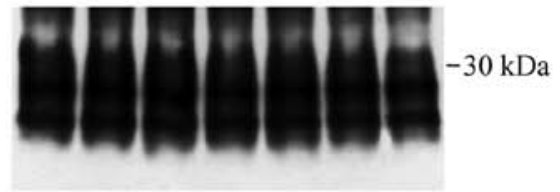

B

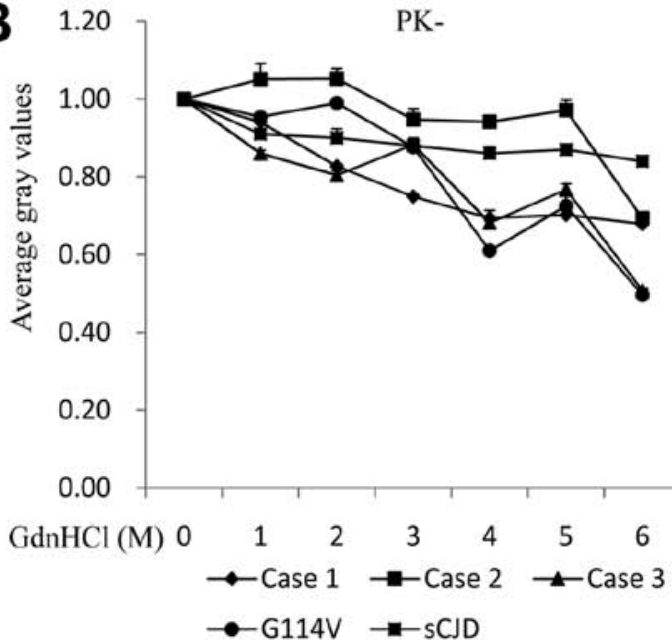

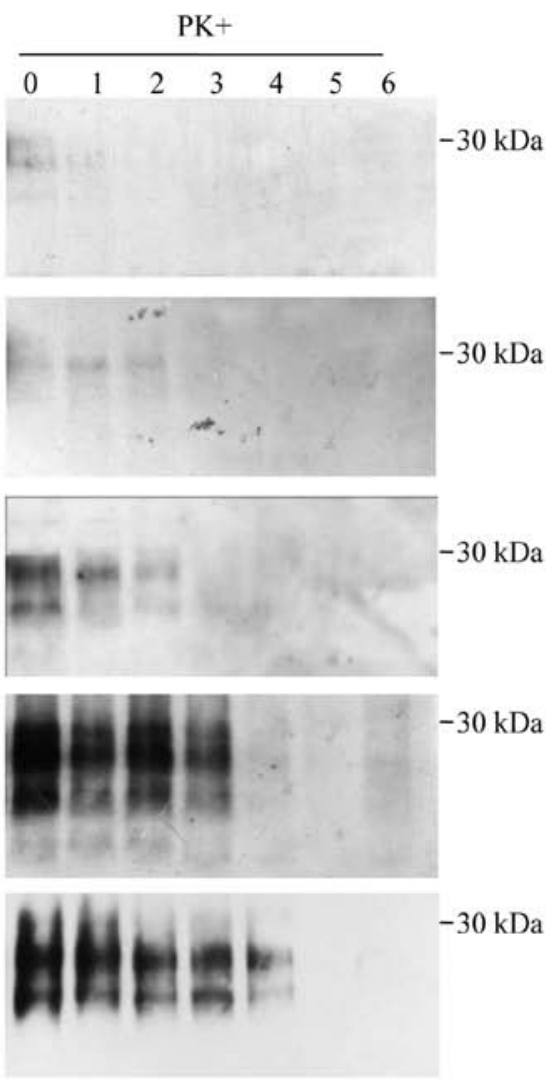

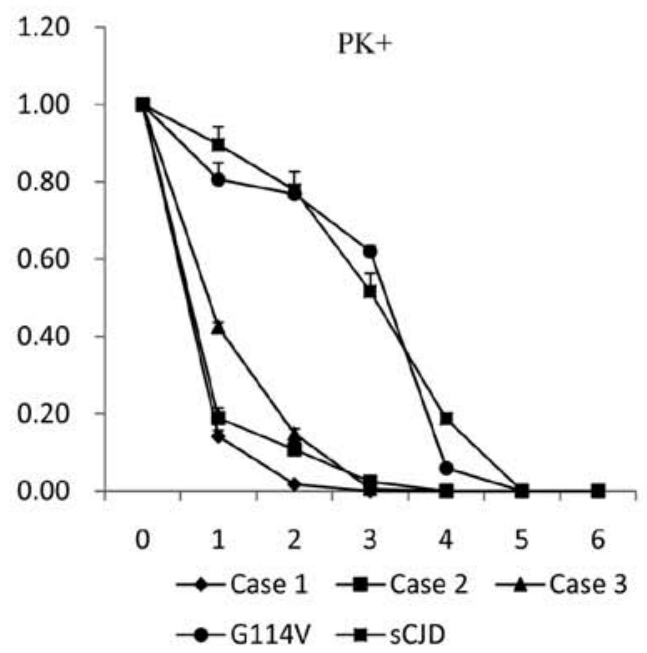

Figure 3. Conformational stability of $\mathrm{PrP}^{\mathrm{Sc}}$ from postmortem FFI, G114V gCJD and sCJD brains. (A) Western blot analyses of total PrP (left panels) and PrPs (right panels) with mAb 3F4. (B) Quantitative analyses of each gray numerical value of the signals of total PrP (left graph) and PrPsc (right graph). The values are calculated following normalization with those of the individual preparation without GdnHCl treatment and presented as the means \pm SD.

PK digestion, in which the signal intensities in the thalamus were slightly weaker than in the cortex regions (Fig. 2A, upper row). To address the deposits of PK-resistant $\operatorname{PrP}\left(\mathrm{PrP}^{\mathrm{Sc}}\right)$ in the brains, various tissue homogenates were treated with $20 \mu \mathrm{g} / \mathrm{ml}$ PK. Clear $\mathrm{PrP}^{\mathrm{Sc}}$ signals were detected in the brains of Cases 2 and 3, particularly in the regions of the thalamus, frontal and parietal lobes, while almost no $\mathrm{PrP}^{\mathrm{Sc}}$ was found in the brain tissues of Case 1 (Fig. 2A, middle row). In contrast to the profiles of type-1 $\mathrm{PrP}^{\mathrm{Sc}}$ in a G114V gCJD and an sCJD with M129 homozygote which contained predominant monoglycosylated $\mathrm{PrP}^{\mathrm{Sc}}$, both detectable $\mathrm{PrP}^{\mathrm{Sc}}$ signals in Cases 2 and 3 showed predominant diglycosylated $\operatorname{PrP}^{\mathrm{Sc}}$ patterns (Fig. 2B), which is more like that of type-2B $\operatorname{PrP}^{\mathrm{Sc}}(8)$.
To assess the PK-resistance of $\mathrm{PrP}^{\mathrm{Sc}}$ in the brains, the homogenates of the thalamus region of the three cases were subjected to treatments with a serial concentration of PK. Western blot analyses showed very weak $\mathrm{PrP}^{\mathrm{Sc}}$ signals in the preparations of Case 1 below $20 \mu \mathrm{g} / \mathrm{ml} \mathrm{PK}$, but obvious $\mathrm{PrP}^{\mathrm{Sc}}$ signals in the preparations of Cases 2 and 3 (Fig. 2C). Analyses of the intensities of $\mathrm{PrP}^{\mathrm{Sc}}$ signals revealed that the curve of $\mathrm{PrP}^{\mathrm{Sc}}$ in Case 1 dropped gradually in the reactions from 5 to $10 \mu \mathrm{g} / \mathrm{ml} \mathrm{PK}$ and reached an undetectable level in that of $20 \mu \mathrm{g} / \mathrm{ml} \mathrm{PK}$, while in Cases 2 and 3 the data maintained relative high levels in the preparations with low PK concentrations (from 5 to $15 \mu \mathrm{g} / \mathrm{ml}$ ) and dropped down to almost baseline in $25 \mu \mathrm{g} / \mathrm{ml}$ PK (Fig. 2D). 

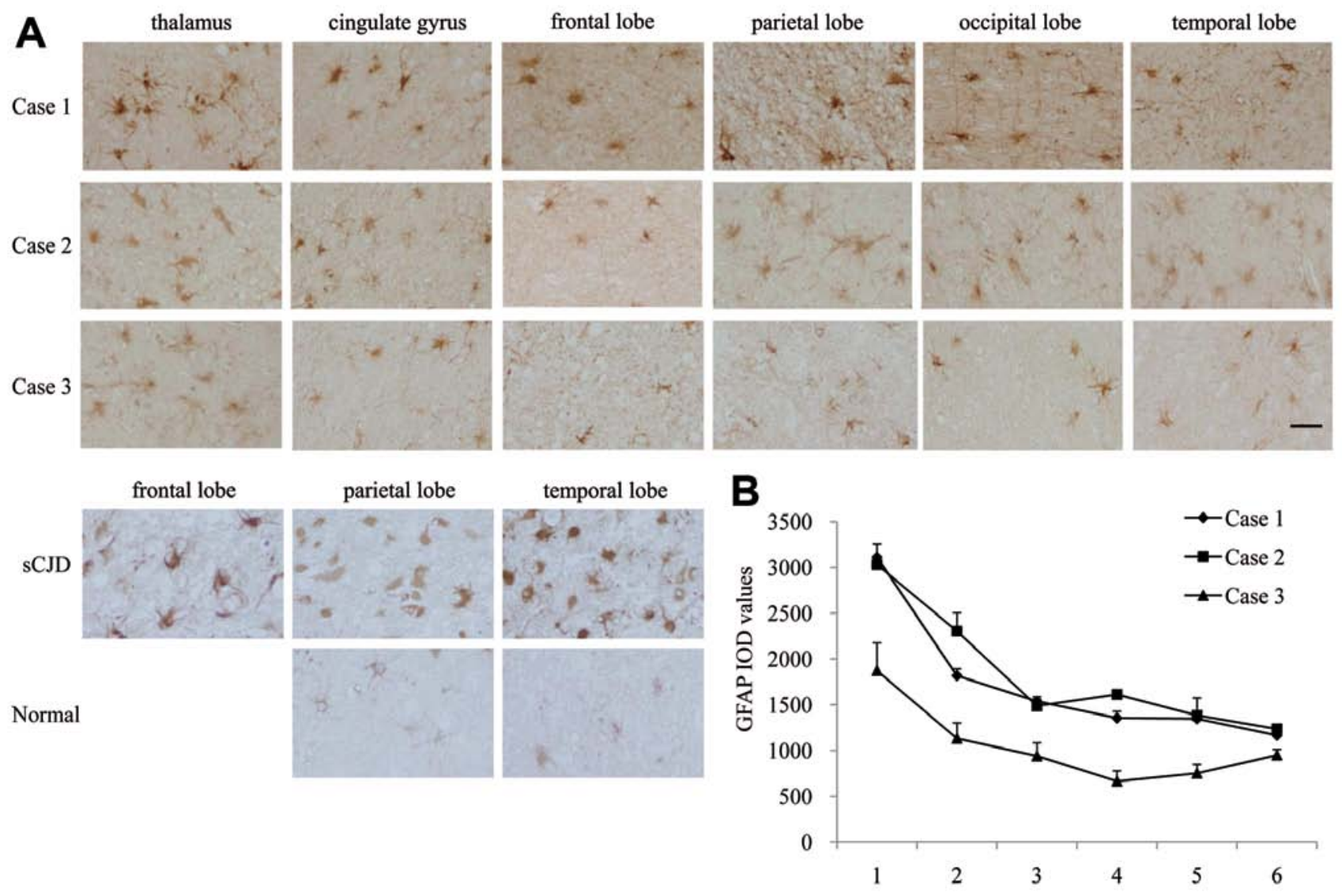

Normal

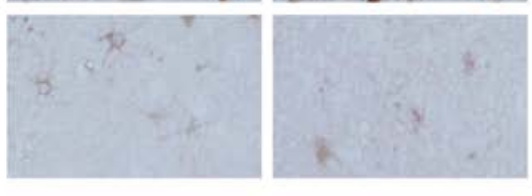

Figure 4. IHC assays of GFAP positive cells in 3 FFI patients. (A) IHC assays under light microscopy. Brain sections of an sCJD and a healthy human were used as controls. Scale bar, $20 \mu \mathrm{m}$. (B) The IOD values determined for various regions of the brains of the 3 cases. The data are expressed as the means \pm SD. The numbers 1-6 along the X-axis represent the thalamus, cingulate gyrus, frontal lobe, parietal lobe, occipital lobe and temporal lobe, respectively.

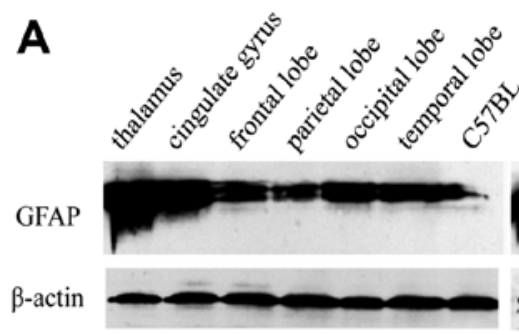

Case 1

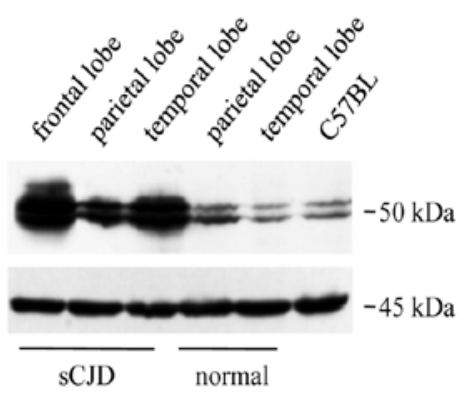

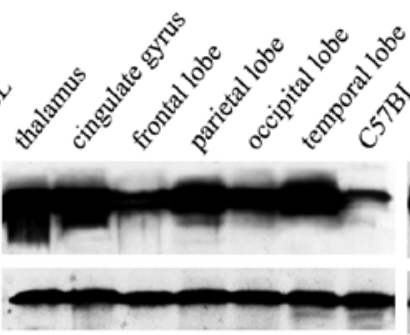

Case 2

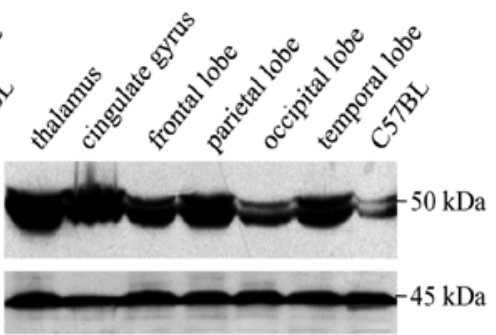

Case 3

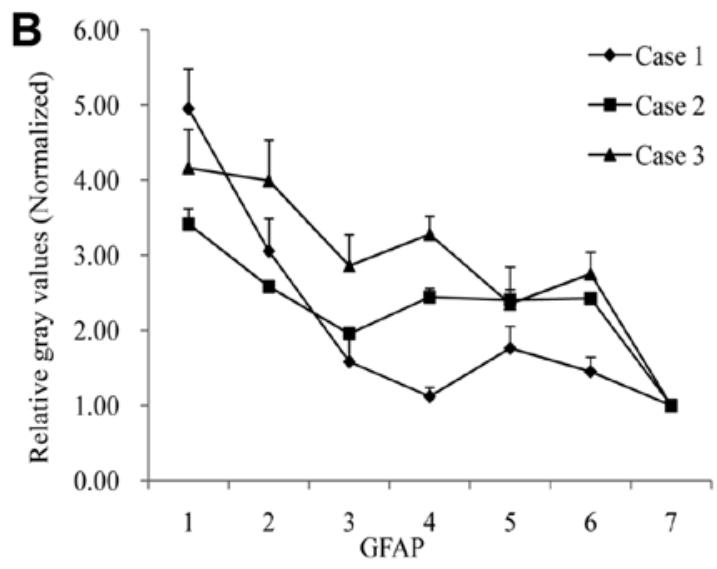

Figure 5. Determination of the relative GFAP amounts in 3 FFI patients. (A) Western blot analyses. Brain tissues of an sCJD and a healthy human were used as controls. (B) The relative gray values of the GFAP signals analyzed by western blot analyses following normalization with the pooled brain homogenates of C57BL mice. The numbers 1-6 along the X-axis represent the thalamus, cingulate gyrus, frontal lobe, parietal lobe, occipital lobe and temporal lobe, respectively. 

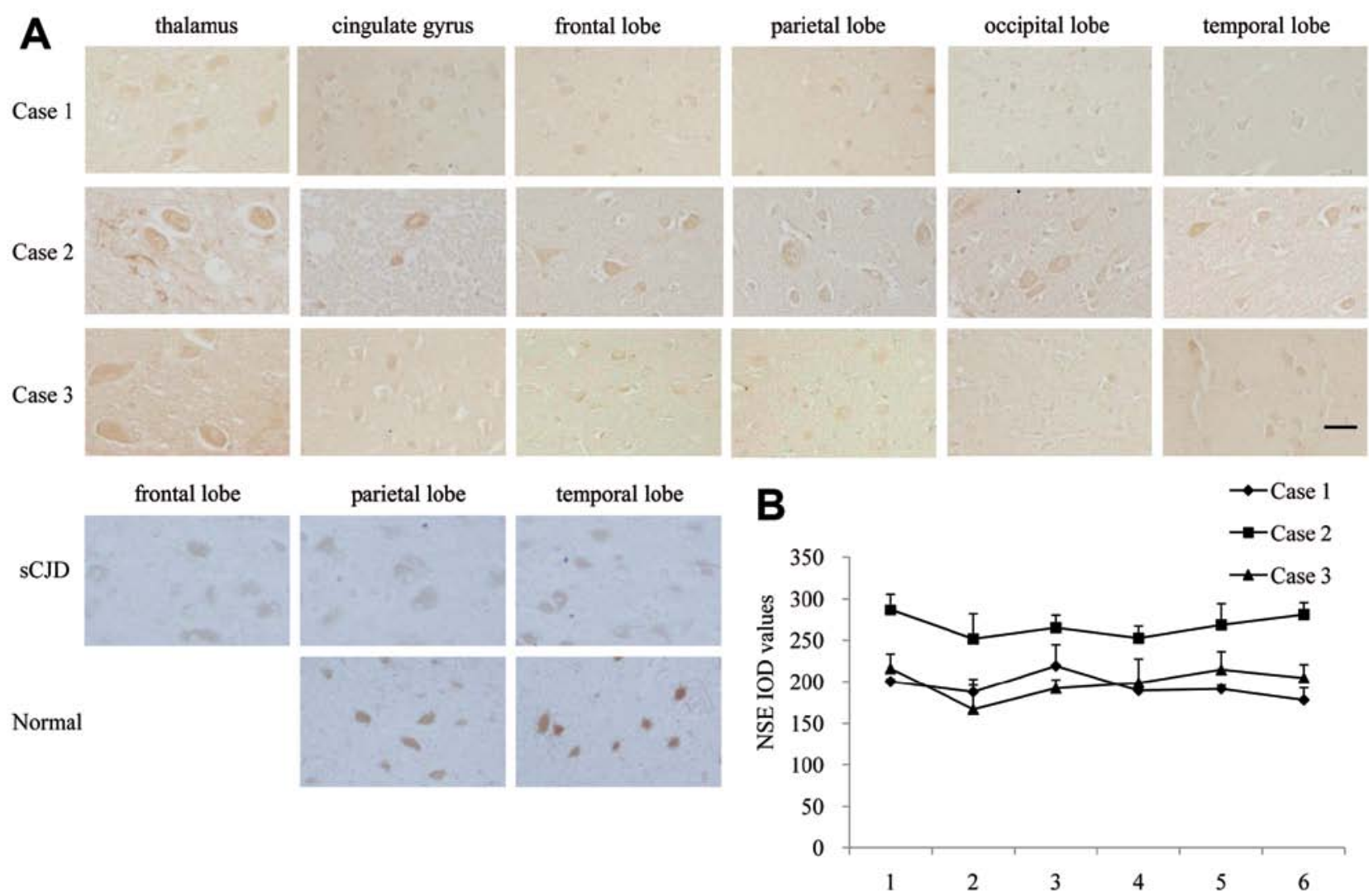

Figure 6. IHC assays of NSE positive cells in 3 FFI patients. (A) IHC assays under light microscopy. Brain sections of an sCJD and a healthy human were used as controls. Scale bar, $20 \mu \mathrm{m}$. (B) The IOD values of various regions of 3 cases. Data are expressed as the means \pm SD. The numbers 1-6 along the X-axis represent the thalamus, cingulate gyrus, frontal lobe, parietal lobe, occipital lobe and temporal lobe, respectively.

Conformational stabilities of $\operatorname{PrP}^{S c}$ from FFI, $g$ CJD and $s C J D$. To test the conformational stabilities of $\operatorname{PrP}^{\mathrm{Sc}}$ in the brains of FFI cases following exposure to $\mathrm{GdnHCl}$, equal amounts of homogenates from the thalamus region of FFI cases, a G114V gCJD patient and an SCJD patient, were incubated with increasing concentrations of $\mathrm{GdnHCl}$. Western blot analyses revealed that the total PrP signals (without treatment of PK) were comparable among five tested samples and decreased slightly along with the increase of $\mathrm{GdnHCl}$ (Fig. 3A and B, left). When the GdnHCl-treated brain samples were subsequently exposed to $20 \mu \mathrm{g} / \mathrm{ml} \mathrm{PK}$, the $\operatorname{PrP}^{\mathrm{Sc}}$ signals in the FFI samples were generally markedly weaker than those shown for the G114V gCJD and sCJD samples. Extremely weak $\operatorname{PrP}^{\mathrm{Sc}}$ signals were observed in the preparations of FFI Case 1 treated with 0 and $1 \mathrm{M} \mathrm{GdnHCl}$, while weak but repeatedly observed $\mathrm{PrP}^{\mathrm{Sc}}$ signals were seen in FFI Cases 2 and 3 treated with 0,1 and $2 \mathrm{M} \mathrm{GdnHCl}$ (Fig. 3A, right). Analyses of the curves of the $\mathrm{PrP}^{\mathrm{Sc}}$ gray values along with the increase of $\mathrm{GdnHCl}$ showed the obviously different patterns among those CJD subtypes, that $\mathrm{PrP}^{\mathrm{Sc}}$ signals of G114V gCJD and sCJD cases possessed stronger conformational stabilities in $\mathrm{GdnHCl}$, while the $\mathrm{PrP}^{\mathrm{Sc}}$ in the brains of the FFI cases possessed less stable conformational topology (Fig. 3B, right).

Marked astrogliosis in the thalamus of FFI cases. To compare the astrogliosis in various brain regions of the 3 FFI cases, the brain slides from six regions of each patient were immunohistochemically stained with a GFAP-specific antibody. Obvious
GFAP-positive stained astrocyte-like cells and fibril-like structures were observed in all tested slides, and the thalamus regions of the 3 patients appeared more severely affected (Fig. 4A). The IOD values of GFAP staining for all three FFI cases were the highest in the thalamus. Comparatively, Case 3 displayed overall lower IOD values in each brain region when compared with the other 2 cases (Fig. 4B). Furthermore, various brain homogenates were employed into GFAP-specific western blot analyses. Fig. 5A illustrates the GFAP-specific signals in all preparations at the position of $M r$. Fifty GFAP signals in the thalamus of the three cases were markedly stronger than those of the individual cortex regions. Analyses of the relative gray values of GFAP signals following normalization with those of the controls of the normal human brain and the pooled mice brain homogenates revealed similar patterns of the GFAP distributions to those the IHC assay showed in the brain regions (Fig. 5B), indicating more astrogliosis in the regions of the thalamus of the 3 FFI cases.

Neuron loss in the different brain regions of FFI cases. Slides of 6 brain regions from 3 FFI cases were immunohistologically stained with an NSE-specific mAb. Large elliptic positive-stained cells were observed in all tested slides (Fig. 6A). Quantification of histochemical staining for NSE showed almost identical levels among the 6 tested regions for each patient, while Case 2 was relatively higher (Fig. 6B). In NSE-specific western blot analyses, the $45 \mathrm{kDa}$-large band was detected in all tested samples, showing similar signal intensi- 


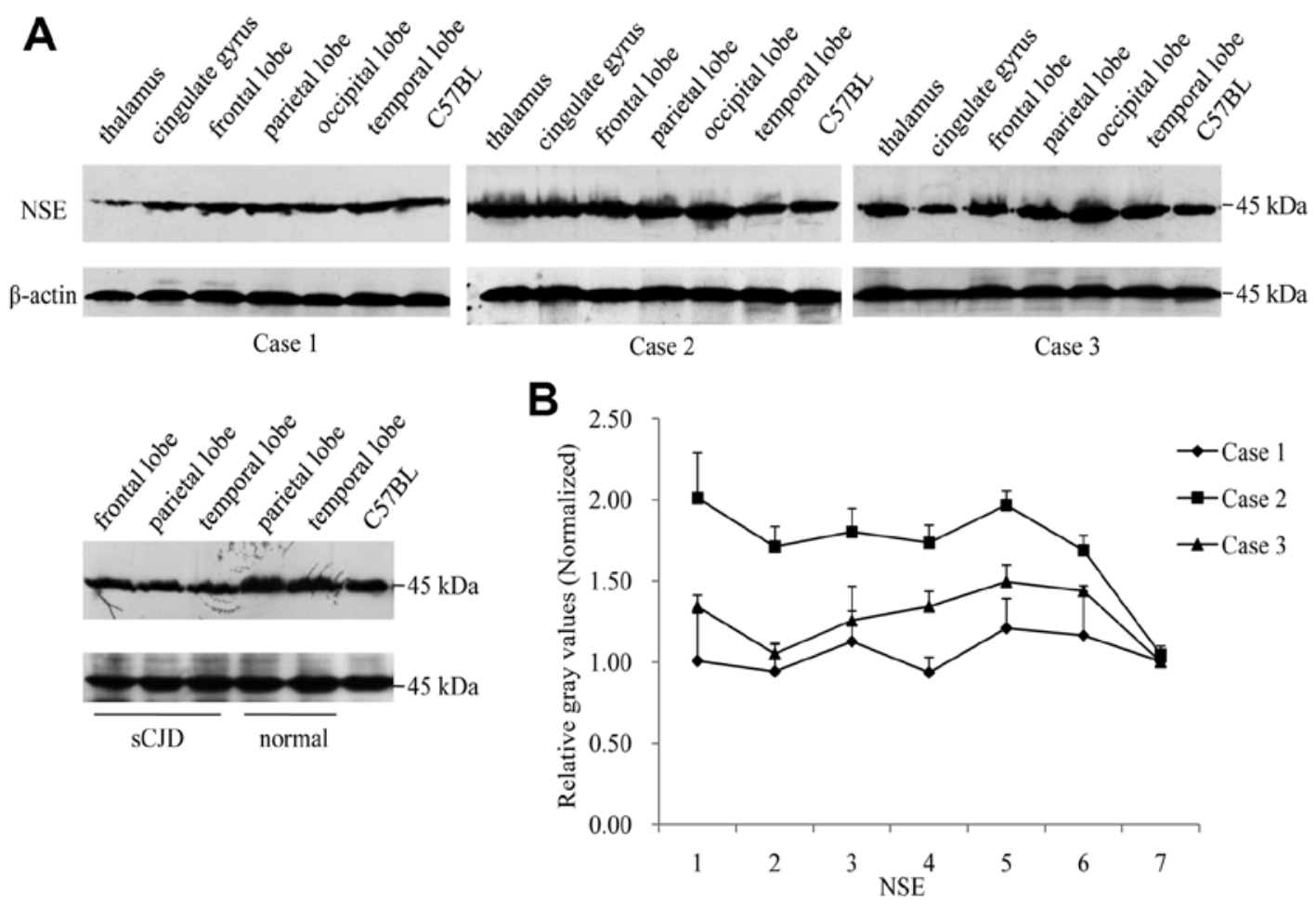

Figure 7. Determination of the relative NSE amounts in 3 FFI patients. (A) Western blot analyses. Brain tissues of an sCJD and a healthy human were used as controls. (B) The relative gray values of the NSE signals analyzed by western blot analyses following normalization with the pooled brain homogenates of C57BL mice. The numbers from 1 to 6 in the X-axis represent the thalamus, cingulate gyrus, frontal lobe, parietal lobe, occipital lobe and temporal lobe, respectively.

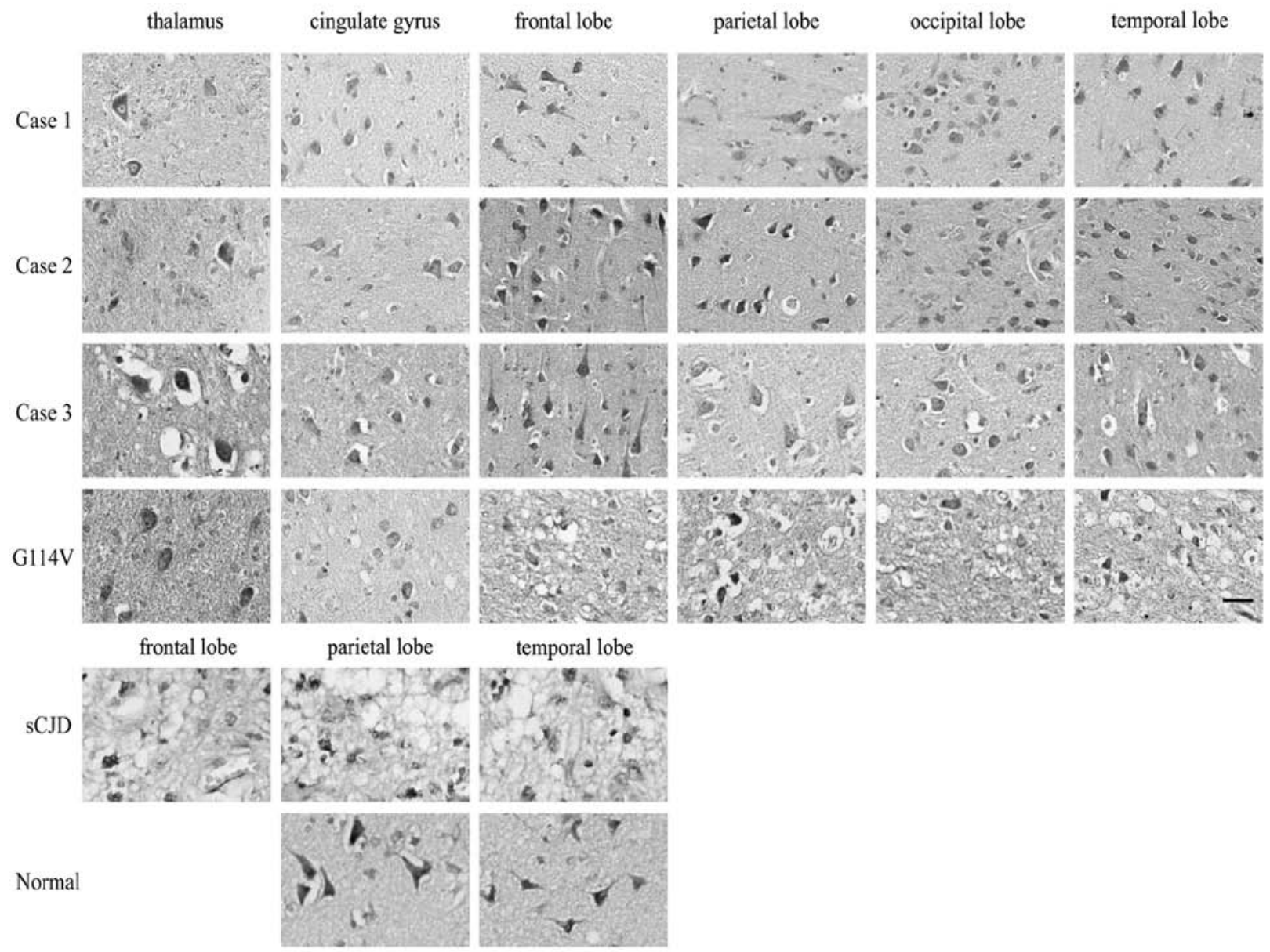

Figure 8. Nissl staining of the six brain regions of 3 FFI patients and a G114V gCJD patient. Brain sections of an sCJD and a healthy human were used as controls. Scale bar, $20 \mu \mathrm{m}$. 

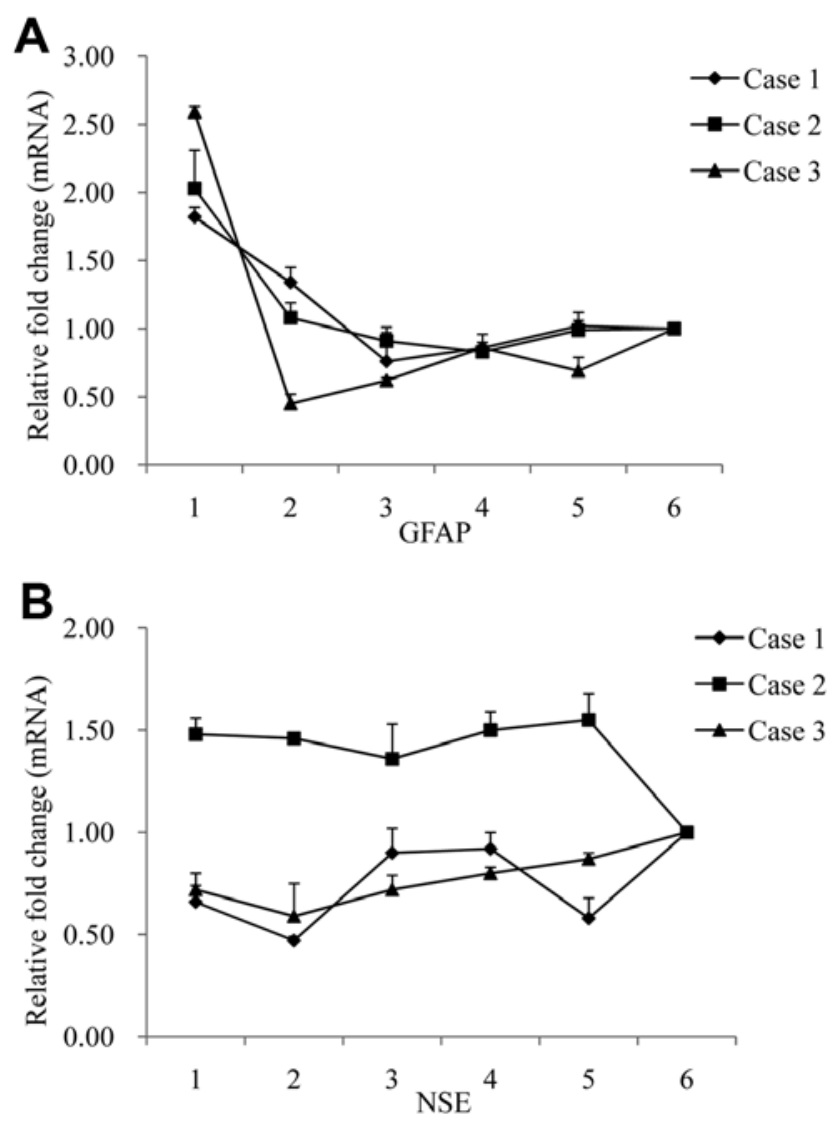

Figure 9. Comparison of the transcriptional levels of (A) GFAP and (B) NSE specific mRNA in the 6 brain regions of 3 FFI patients. Y-axis represents mRNA fold change compared to the temporal lobe of each case. The numbers from 1 to 6 in the $\mathrm{X}$-axis represent the thalamus, cingulate gyrus, frontal lobe, parietal lobe, occipital lobe and temporal lobe, respectively.

ties among various FFI cases (Fig. 7A). Relative gray values of NSE signals in each preparation following equilibration with those of the normal human and mice brain homogenates illustrated comparable data among various brain regions, in which the data of Case 2 were higher (Fig. 7B).

Slides of 6 brain regions from three FFI cases and a G114V gCJD were assayed by Nissl staining. It revealed less purpleblue stained Nissl bodies in the regions of the thalamus of the three FFI cases accompanied by vacuoles, compared with the observations in the same region of G114V gCJD (Fig. 8). By contrast, more severe spongiform degenerations were observed in the 4 cortex lobes of the G114V gCJD case than in the 3 FFI cases, accompanied by obvious pyknotic nuclei (Fig. 8). This finding suggests more severe neuronal damage in the thalamus region of FFI.

Transcriptions of GFAP and NSE in brains of FFI cases. To evaluate the transcriptional levels of GFAP and NSE-specific mRNAs in various brain regions, total RNAs extracted from 6 brain regions of 3 FFI cases were subjected to qRT-PCRs for GFAP and NSE, respectively, using the amplification of GAPDH mRNA as the endogenous control. Compared to the 4 cortex regions, the thalamus expressed by far the highest relative quantities of GFAP-specific mRNA ( $\left.2^{-\Delta \Delta C t}\right)$ (Fig. 9A). There was little difference in the relative expression of NSE specific mRNAs $\left(2^{-\Delta \Delta C t}\right)$ among various brain regions of each patient, although the values in Case 2 appeared higher than in the other 2 cases (Fig. 9B). The data suggested that the transcriptions of GFAP and NSE in brain tissues of FFI patients correlated positively with the levels of the proteins.

\section{Discussion}

In this study, we investigated evidence for astrogliosis and neuronal loss in various brain regions from postmortem samples obtained from 3 Chinese FFI cases. Based on the semi-quantitative methodologies of IHC and western blot analyses for GFAP and NSE, the brains of the 3 FFI cases displayed similar neuropathological patterns, whereby a more active gliosis in the regions of the thalamus was found compared to the cortex regions.

GFAP is restrictively expressed by astrocyte, and is commonly used as a special biomarker for astrocyte. Astrogliosis is a basic pathogenic sign often associated with CNS injuries (15), showing increased expression of GFAP in brain tissues. Severe astrogliosis in the thalamus is significant for the FFI-related pathologic pattern, which is distinct from other types of human prion diseases $(9,16)$. Our data here provide evidence that not only higher numbers of GFAPstained cells, but also more deeply GFAP-stained and larger astrocytes are observed in the thalamus of FFI patients. Our observations emphasize again the unique pathogenesis of FFI, caused by the existence of the PRNP allele of the mutation of D178N with M129M homozygote.

Neuronal loss is another pathological feature of prion diseases, although it is not the specific one $(3,4,6,17)$. From the Nissl staining, which presents the situation of the neuronal structural destruction, it appears that more severe neuronal damage occurs in the thalamus of the FFI cases than in the G114V gCJD case, who had sCJD-like clinical presentations and neuropathological changes (9). However, we were unable to find significant differences in the number of NSE-stained cells or degree of NSE gene/protein expression among the various brain regions of individual FFI patients. Furthermore, since the amount of NSE-positive cells or signals in the brain of the Case 2 patient, who was 26 years old at onset, tested repeatedly higher than in the other 2 patients, who were in their 50's at onset, an age-related factor is possible.

Our study here illustrates again the common characteristics of FFI, presence of little or no $\mathrm{PrP}^{\mathrm{Sc}}$ in brain tissues with less PK-resistance and unstable in conformation when exposed to $\mathrm{GdnHCl}$, possibly indicating a special topology of PrP with D178N mutation and M129M homozygous. In line with several other studies $(2,4,18)$, our data reveals whether or not deposition of $\mathrm{PrP}^{\mathrm{Sc}}$ seems to significantly affect the neuropathological changes and the clinical manifestations of FFI; it may also highlight that the large plaques or severe aggregations of $\mathrm{PrP}^{\mathrm{Sc}}$ are not the major component for neurotoxicity in prion diseases (19).

In CJD patients, some disease-related genes are usually continually transcribed in the CNS tissues, even at the final stage of illness. We previously demonstrated that PrP mRNAs are continuously transcribed in the brain tissues of a G114V gCJD, which is speculated to supply PrPC as the substrates for $\mathrm{PrP}^{\mathrm{Sc}}$ replication (9). Active transcription of GFAP in 
brains, especially in the thalamus, may reflect an intense reactive gliosis against the progressively severe brain injury. Identification of NSE-related mRNAs in FFI brains during their terminal stage may indicate the presence of non-functional neurons or neuron networks.

To date, more than 56 mutations in PrP have been identified as being involved in the development of human genetic TSEs with a variety of geographical distributions and frequencies (16). Although the genetic tendencies, the pathologic involvements, the clinical manifestations and the laboratory features may vary, no other TSE induces such prominent thalamic dysfunction characterized by insomnia and dysautonomia as do TSEs containing a D178N mutation with M129M polymorphism. Why FFI-associated PrP mutant has tropisms for the thalamus remains unknown. Other human genetic prion diseases also show region-specific damage, such as GSS in the cerebellum (20). Further studies into the role of PrP, as well the local microenvironment, are required in order to better understand the pathogenesis of this mysterious disease.

\section{Acknowledgements}

We thank Dr Dennis J. Grab (Johns Hopkins University) for reading our manuscript prior to submission. This study was supported by the China Mega-Project for Infectious Disease (2011ZX10004-101), the Young Scholar Scientific Research Foundation of China CDC (2012A102), and the SKLID Development Grant (2012SKLID102 and 2011SKLID211).

\section{References}

1. Goldfarb LG, Petersen RB, Tabaton M, et al: Fatal familial insomnia and familial Creutzfeldt-Jakob disease: disease phenotype determined by a DNA polymorphism. Science 258: 806-808, 1992.

2. Medori R, Montagna P, Tritschler HJ, et al: Fatal familial insomnia: a second kindred with mutation of prion protein gene at codon 178. Neurology 42: 669-670, 1992.

3. Lugaresi E, Medori R, Montagna P, et al: Fatal familial insomnia and dysautonomia with selective degeneration of thalamic nuclei. N Engl J Med 315: 997-1003, 1986.

4. Manetto V, Medori R, Cortelli P, et al: Fatal familial insomnia: clinical and pathologic study of five new cases. Neurology 42 : 312-319, 1992.

5. Ayuso Blanco T, Urriza Mena J, Caballero Martinez C, Iriarte Franco J, Munoz R and Garcia-Bragado F: Fatal familiar insomnia: clinical, neurophysiological and histopathological study of two cases. Neurologia 21: 414-420, 2006 (In Spanish).
6. Parchi P, Petersen RB, Chen SG, et al: Molecular pathology of fatal familial insomnia. Brain Pathol 8: 539-548, 1998.

7. Brown P, Kenney K, Little B, et al: Intracerebral distribution of infectious amyloid protein in spongiform encephalopathy. Ann Neurol 38: 245-253, 1995.

8. Shi XH, Han J, Zhang J, et al: Clinical, histopathological and genetic studies in a family with fatal familial insomnia. Infect Genet Evol 10: 292-297, 2010.

9. Shi Q, Zhang BY, Gao C, et al: The diversities of $\operatorname{PrP}(\mathrm{Sc})$ distributions and pathologic changes in various brain regions from a Chinese patient with G114V genetic CJD. Neuropathology 32: $51-59,2012$

10. Thackray AM, Hopkins L, Spiropoulos J and Bujdoso R: Molecular and transmission characteristics of primary-passaged ovine scrapie isolates in conventional and ovine PrP transgenic mice. J Virol 82: 11197-11207, 2008.

11. Zheng Q, Zhu D, Bai Y, Wu Y, Jia J and Hu Y: Exercise improves recovery after ischemic brain injury by inducing the expression of angiopoietin-1 and Tie-2 in rats. Tohoku J Exp Med 224: 221-228, 2011.

12. Zhang J, Chen L, Zhang BY, et al: Comparison study on clinical and neuropathological characteristics of hamsters inoculated with scrapie strain $263 \mathrm{~K}$ in different challenging pathways. Biomed Environ Sci 17: 65-78, 2004.

13. Ruifrok AC, Katz RL and Johnston DA: Comparison of quantification of histochemical staining by hue-saturationintensity (HSI) transformation and color-deconvolution. Appl Immunohistochem Mol Morphol 11: 85-91, 2003.

14. Abdulmawjood A, Schonenbrucher $\mathrm{H}$ and Bulte $\mathrm{M}$ : Novel molecular method for detection of bovine-specific central nervous system tissues as bovine spongiform encephalopathy risk material in meat and meat products. J Mol Diagn 7: 368-374, 2005.

15. Eng LF and Ghirnikar RS: GFAP and astrogliosis. Brain Pathol 4: 229-237, 1994.

16. Pastore M, Chin SS, Bell KL, et al: Creutzfeldt-Jakob disease (CJD) with a mutation at codon 148 of prion protein gene: relationship with sporadic CJD. Am J Pathol 167: 1729-1738, 2005.

17. Spacey SD, Pastore M, McGillivray B, Fleming J, Gambetti P and Feldman H: Fatal familial insomnia: the first account in a family of Chinese descent. Arch Neurol 61: 122-125, 2004.

18. Almer G, Hainfellner JA, Brucke T, et al: Fatal familial insomnia: a new Austrian family. Brain 122: 5-16, 1999.

19. Faucheux BA, Morain E, Diouron V, et al: Quantification of surviving cerebellar granule neurones and abnormal prion protein $\left(\mathrm{PrP}^{\mathrm{Sc}}\right)$ deposition in sporadic Creutzfeldt-Jakob disease supports a pathogenic role for small $\mathrm{PrP}^{\mathrm{Sc}}$ deposits common to the various molecular subtypes. Neuropathol Appl Neurobiol 37: 500-512, 2011.

20. Kretzschmar HA, Kufer P, Riethmuller G, DeArmond S, Prusiner SB and Schiffer D: Prion protein mutation at codon 102 in an Italian family with Gerstmann-Straussler-Scheinker syndrome. Neurology 42: 809-810, 1992. 